\title{
Premedication With Oral Pregabalin for the Prevention of Acute Postsurgical Pain in Coronary Artery Bypass Surgery
}

\author{
MohsenZiyaeifard ${ }^{1}$;MohammadJavad Mehrabanian ${ }^{2}$;SeyedehZahraFaritus ${ }^{1, *} ;$ Mehrdad $^{2}$ \\ Khazaei Koohpar ${ }^{2}$; Rasool Ferasatkish ${ }^{1}$; Heidar Hosseinnejad ${ }^{3}$; Mohammadreza \\ Mehrabanian $^{4}$ \\ ${ }^{1}$ Department of Anesthesiology, Shahid Rajaie Cardiovascular Medical and Research Center, Tehran, Iran \\ Department of Cardiac Anesthesia, Shahid Rajaie Cardiovascular Medical and Research Center, Tehran, IR Iran. \\ ${ }^{3}$ Department of Anesthesia, Shahrood Branch of Islamic Azad University, Shahrood, Iran \\ 4 Department of Neurosurgery, Shahid Sadoughi University of Medical Sciences, Yazd, Iran \\ *Corresponding author: Seyedeh Zahra Faritus, Department of Anesthesiology, Shahid Rajaie Cardiovascular Medical and Research Center, Tehran, Iran. Tel: +98-2123921, Fax: +98- \\ 2122042026, E-mail: s.faritous@yahoo.com \\ Received: October 26, 2014; Revised: November 27, 2014; Accepted: December 4, 2014
}

\begin{abstract}
Background: For coronary artery bypass grafting (CABG) sternotomy should be performed. The pain after surgery is severe and requires medical intervention. Use of the analgesics is limited by their side effects and studies suggest that prevention with some medications before surgery is effective in controlling the postoperative pain.

Objectives: We investigated the efficacy of pregabalin administration before surgery in the treatment of acute postoperative pain after CABG surgery.

Patients and Methods: Sixty patients indicated for elective CABG surgery were randomly allocated to two groups. One group received placebo and the other received $150 \mathrm{mg}$ of oral pregabalin before surgery. Heart rates, blood pressure, respiratory rate, intensive care unit (ICU) stay duration, morphine consumption, and pain score according to the visual analog scale (VAS) were measured and recorded at 4, 12 , and 24 hours of surgery.

Results: Pregabalin consumption did not alter hemodynamic parameters and was safe in patients after CABG. Its consumption was associated with significant reduction in the pain score (P values were $0.035,0.026$, and 0.047 respectively at 4,12 , and 24 hours of surgery). Its use was not associated with changes in the morphine consumption at 4,12 , and 24 hours of surgery $(\mathrm{P}>0.05)$.

Conclusions: Premedication with studied dose of pregabalin is effective for the prevention of postoperative pain in patients after CABG and has no adverse effects. Trials with other treating schedule and doses of the drug should be performed to determine the best treatment plan.
\end{abstract}

Keywords:Pregabalin; Pain Management; Acute Pain; Morphine; Coronary Artery Bypass

\section{Background}

Atherosclerotic narrowing of coronary arteries is a common adverse condition that requires medical attention and in many cases, coronary artery bypass grafting (CABG) is indicated for high-risk patients (1-7). In CABGs, patients have severe pain during and after surgery due to sternotomy. Acute pain after cardiac surgery might be visceral, musculoskeletal, or neurogenic in origins requiring medical attention $(8,9)$. Acute pain after surgery is an undesirable outcome of CABG that might turn into persistent and debilitating postoperative chronic pain (10). It directly correlates with prolonged surgery. In a study by Lahtinen et al., $49 \%, 78 \%$, and $62 \%$ of patients experienced severe pain at rest, during coughing, and on movement, respectively (11). According to the same study, $31 \%$ of patients were having pain upon movement even one year after surgery. Management of the condition is difficult and most of the patients receive opioids for pain relief (9). Due to several known adverse effects of opioids, efforts are being made to replace existing drugs with newer ones with fewer adverse effects and to develop novel approaches for reducing postsurgery pain (12).

The anticonvulsant pregabalin is indicated for the treatment of peripheral neuropathic pain. Similar to gabapentin, pregabalin is an analog of neurotransmitter gamma-aminobutyric acid (GABA) (13). It mainly acts through binding on alpha-2 and delta receptors and acting as antihyperalgesic agent (14). Pregabalin delays or offsets the sensitization of dorsal horn neurons, possibly leading to augmentation of surgical stimulation that affects changes in the central and peripheral nervous system. Recent studies on pregabalin use for reducing postoperative pain have revealed its beneficial effects on the prevention of pain as well as altering the neuropathic pain incidence. A systematic review by Clarke et al. on pregabalin use in postoperative pain has confirmed its safety and effectiveness (15). However, another systematic review by Chaparro et al. concluded that enough evidences are not available to make conclusion whether gabapentin is suit-

Copyright (c) 2015, Iranian Society of Regional Anesthesia and Pain Medicine(ISRAPM).This is an open-access article distributed under the terms of the Creative Commons Attribution-NonCommercial 4.0 International License (http://creativecommons.org/licenses/by-nc/4.0/) which permits copy and redistribute the material just in noncommercial usages, provided the original work is properly cited. 
able for prevention of postsurgical pain (10). In addition, optimal dose and duration of the treatment cannot be recommended because of the heterogeneity of the trials.

\section{Objectives}

The aim of the current study was to investigate whether premedication with pregabalin was effective in the treatment of acute postoperative pain after CABG.

\section{Patients and Methods}

\subsection{Study Design}

A total of 60 patients referred to the Rajaie Cardiovascular, Medical and Research Center, Tehran, Iran. for elective CABG were recruited in this randomized, controlled, double-blinded trial. Patients and examining physicians were unaware of the intervention used for the patient during study. Patients were randomly allocated to two groups: one group was set as control and the second received pregabalin. For randomization purpose, we used the http://www.randomizer.org online software. According to Sunder et al. study in 2012 that assessed pregabalin effect on post-op pain, he found VAS in pregabalin and control group $2.02 \pm 57$ and $2.39 \pm 0.61$, respectively by using online sample size calculator http://www.stat.ubc. ca/ rollin/stats/ssize/n2.html we calculated 29 cases in each group. Finally, we chose the sample size of 30 in each group and a total of 60 patients.

Patients were assigned numbers from one to 60 and each was randomly assigned either into control or pregabalin groups as defined randomly by the software. Patients older than 20 years old who were planned for elective CABG with Laryngeal view grade from 1 to 3, according to the American Society of Anesthesiologists (ASA) guideline on management of the difficult airway (16), were included in this study. The anesthesia and surgical techniques were the same for all the patients.

Patients with liver or renal dysfunction, metabolic disorders, and left bundle branch block (LBBB) were excluded from study. In addition, patients with indications of emer- gency surgical operation, those using opioids, patients with a history of drug sensitivity or seizures, smokers, and those with ejection fraction $(E F)<35 \%$ were excluded. Patients signed informed consent form. Study protocol was approved by Institutional Ethics Review Board of the Rajaei Heart Center. Protocol of the study was in compliance with Helsinki declaration on ethical principles for medical research involving human subjects (17).

\subsection{Treatment Plan and Outcome Measures}

Patients in pregabalin and control groups respectively received single dose of 150-mg pregabalin capsules (LYRICA, Pfizer Inc, Germany) and placebo two hours before surgery. Severity of pain after 4,12 and 24 hours were measured in patients by a visual analog scale (VAS) and recorded (18). Patients having VAS scores $>3$ received 0.1 $\mathrm{mg} / \mathrm{kg}$ of intravenous morphine up to $8 \mathrm{mg}$. Background data including arterial blood pressure, heart rate, respiratory rate, and time of extubation were recorded.

\subsection{Statistical Analysis}

Numerical data were expressed as mean \pm standard deviation (SD). As the data showed normal distribution pattern by Kolmogorov-Smirnov test as well as homogeneity of variance, group comparisons were made by Student's t test. Chi square test was used to examine differences between qualitative data. In all comparisons, statistical significance levels were considered at $\mathrm{P}<0.05$.

\section{Results}

Overall, 60 patients were recruited for this study among which 10 (16.6\%) were female. Rate of females in pregabalin and control groups were similar and identical to its frequency in total patients. Mean age of control and pregabalin groups were $57.9 \pm 8.6$ and $54.7 \pm 8.3$ years, respectively $(\mathrm{P}>0.05)$. Although our exclusion criteria was the patients age $>20$ years, the age of patients ranged from 35 to 70 years due to occurrence of the coronary artery events at older ages.

\begin{tabular}{|c|c|c|c|}
\hline Variable & Pregabalin Group, $\mathbf{n}=\mathbf{3 0}$ & Control Group, $\mathbf{n}=\mathbf{3 0}$ & P Value \\
\hline \multicolumn{4}{|l|}{ Heart rate } \\
\hline At 4 hours postsurgery & $95.1 \pm 8.2$ & $95.0 \pm 7.6$ & 0.93 \\
\hline At 12 hours postsurgery & $88.5 \pm 7.8$ & $86.4 \pm 8.2$ & 0.32 \\
\hline At 24 hours postsurgery & $79.1 \pm 9.6$ & $79.4 \pm 8.4$ & 0.92 \\
\hline \multicolumn{4}{|l|}{ Systolic blood pressure } \\
\hline At 4 hours postsurgery & $128.7 \pm 19.2$ & $130.9 \pm 14.6$ & 0.07 \\
\hline At 12 hours postsurgery & $127.6 \pm 18.2$ & $124 \pm 14.1$ & 0.06 \\
\hline At 24 hours postsurgery & $131 \pm 14.7$ & $128 \pm 12.3$ & 0.09 \\
\hline \multicolumn{4}{|l|}{ Diastolic blood pressure } \\
\hline At 4 hours postsurgery & $83.1 \pm 7.2$ & $78.1 \pm 8.7$ & 0.09 \\
\hline At 12 hours postsurgery & $79.3 \pm 6.2$ & $76.1 \pm 8.3$ & 0.1 \\
\hline At 24 hours postsurgery & $77.5 \pm 8.2$ & $75.1 \pm 6.5$ & 0.22 \\
\hline
\end{tabular}


Ziyaeifard M et al.

\begin{tabular}{lccc}
\hline Table 2. Morphine Consumption in Milligrams ${ }^{\mathrm{a}}$ & & \\
\hline Morphine Usage & Pregabalin Group, $\mathbf{n}=\mathbf{3 0}^{\text {Control Group, } \mathbf{n}=\mathbf{3 0}}$ & P Value \\
\hline At $\mathbf{4}$ hours Postsurgery, $\mathbf{~ m g}$ & $5.0 \pm 0.2$ & $4.9 \pm 0.2$ & 0.87 \\
At 12 hours Postsurgery, $\mathbf{m g}$ & $4.6 \pm 0.8$ & $3.8 \pm 0.1 .4$ & 0.11 \\
At 24 hours Postsurgery, $\mathbf{~ m g}$ & $3.0 \pm 0.1 .7$ & $3.1 \pm 0.15$ & 0.94 \\
\hline
\end{tabular}

a Data are presented as Mean \pm SD.

Table 3. Duration of Intensive Care Unit Stay in Study Groups ${ }^{\mathrm{a}, \mathrm{b}}$

\begin{tabular}{lccc}
\hline Variable & Pregabalin Group & Control Group & PValue \\
\hline ICU stay, h & $78.8 \pm 11.5$ & $79.6 \pm 8.1$ & 0.34 \\
\hline a Data are presented as Mean \pm SD. & & &
\end{tabular}

b Pregabalin was administered as $150 \mathrm{mg}$ capsule once a day.

Figure 1. Pain Score of the Patients in Control and Pregabalin Groups.



VAS4,VAS12, and VAS24 are representing pain score according to the visual analog scale (VAS) in patients of each group at 4, 12, and 24 hours postsurgery, respectively.

According to our results, no hemodynamic changes were observed between two groups $(P>0.05)$ (Table 1). Comparison of severity of pain showed significant differences between two groups at 4, 12, and 24 hours of surgery (Figure 1$)(\mathrm{P}<0.05)$. Morphine consumption did not change significantly by using pregabalin. Consumption of morphine was similar between patients receiving pregabalin before treatment and those receiving placebo (Table 2). ICU stay duration was also similar between two groups $(\mathrm{P}>0.05)$ (Table 3$)$.

\section{Discussion}

In the current study, we investigated the effectiveness and safety profile of pregabalin vs control group for the management of acute pain after CABG. Findings of the study showed no significant differences in pain score according to the VAS scale between two groups at 4,12, and 24 hours of surgery. Moreover, using pregabalin did not significantly change the opioid consumption doses.

Although some studies are advocating the use of pregabalin before surgery to reduce postsurgical pain, some others deny its effectiveness $(19,20)$. Studies by Joshi et al. demonstrated that acute and chronic postoperative pain after off-pump CABG in patients receiving pregabalin, $150 \mathrm{mg}$ two hours prior to induction of anesthesia and $75 \mathrm{mg}$ twice daily for two consecutive postoperative days, had significantly lowered pain severity at rest and upon movement in comparison to control group. In addition, pregabalin consumption was associated with $60 \%$ lower rates of tramadol consumption without increasing the extubation time (21). In the current study, we used a single dose of pregabalin (150 mg) before surgery. As our study demonstrated no significant differences between two groups, it appears that premedication by itself is not sufficient to reduce postsurgical pain.

A meta-analysis study by Chaparro et al. for evaluation of the efficacy of systemic drugs for the prevention of chronic pain after surgery by examining the proportion of patients reporting pain three months or more after surgery, could not approve pregabalin preventive effect on postsurgical pain (10). Nonetheless, a similar study by Clarke et al. shows that pregabalin was useful in this case (15). Our study should be added to the evidence that pregabalin might be useful in the management of postoperative pain when administered at single dose of 150 $\mathrm{mg}$ before surgery. According to the meta-analysis by Chaparro et al. (10), ketamine might be more effective in management of acute postsurgical pain. Our study is the first of its kind examining the possibility of using pregabalin to prevent postsurgery pain of CABG. Our trial demonstrated significant differences in pain scores between placebo and pregabalin when administered at single dose of $150 \mathrm{mg}$ before surgery. Considering our findings, findings of other studies and safety profile of the drug, we recommend examining pregabalin effectiveness with different doses and treatment schedules in larger trials to define whether it can be used in management of postoperative pain and to determine a universal dosing and administration schedule.

\section{Acknowledgements}

We are grateful to our patients for cooperation during this study and would like to extend our warmest thanks to our colleagues at Rajaei Heart Center for assisting us in 
conducting this research. We express our special thanks to the study consultant biostatistician, Dr Hooman Bakhshandeh.

\section{Authors' Contributions}

Mohsen Ziyaeifard: conducting study and administration of technical and scientific revision of the article; Seyedeh Zahra Faritus: literature search and clinical analysis; Mohammad Javad Mehrabanian: data interpretation and clinical analysis; Mehrdad Khazaei Koohpar and Rasool Ferasatkish: data interpretation and critical revision of the article; Heidar Hosseinnejad and Mohammadreza Mehrabanian: data collection and manuscript preparation.

\section{References}

1. Eagle KA, Guyton RA, Davidoff R, Edwards FH, Ewy GA, Gardner TJ et al. ACC/AHA 2004 guideline update for coronary artery bypass graft surgery: a report of the American College of Cardiology/ American Heart Association Task Force on Practice Guidelines (Committee to Update the 1999 Guidelines for Coronary Artery Bypass Graft Surgery). Circulation. 2004;110(14):e340-437.

2. Ebrahimi M, Kazemi-Bajestani SM, Ghayour-Mobarhan M, Ferns GA. Coronary artery disease and its risk factors status in iran: a review. Iran Red Crescent Med J. 2011;13(9):610-23.

3. Sarraf-Zadegan N, Sadri G, Malek Afzali H, Baghaei M, Mohammadi Fard N, Shahrokhi S, et al. Isfahan Healthy Heart Programme: a comprehensive integrated community-based programme for cardiovascular disease prevention and control. Design, methods and initial experience. Acta Cardiol. 2003;58(4):309-20.

4. Hadaegh F, Harati H, Ghanbarian A, Azizi F. Prevalence of coronary heart disease among Tehran adults: Tehran Lipid and Glucose Study. East Mediterr Health J. 2009;15(1):157-66.

5. Amani R, Noorizadeh M, Rahmanian S, Afzali N, Haghighizadeh $\mathrm{MH}$. Nutritional related cardiovascular risk factors in patients with coronary artery disease in Iran: a case-control study. Nutr J 2010;9:70.

6. Gorjipour F, Asadi Y, K. Osguei N, Effatkhah M, Samadikuchaksaraei A. Serum level of homocysteine, folate and vitamin-B12 in epileptic patients under carbamazepine and sodium valproate treatment: a systematic review and meta-analysis. Iran Red Crescent Med J. 2013;15(3):249-53.
7. Poursafa P, Kelishadi R, Amin MM, Hashemi M, Amin M. First report on the association of drinking water hardness and endothelial function in children and adolescents. Arch Med Sci. 2014;10(4):746-51.

8. Cogan J. Pain management after cardiac surgery. Semin Cardiothorac Vasc Anesth. 2010;14(3):201-4.

9. Lee W, Yan YY, Jensen MP, Shun SC, Lin YK, Tsai TP, et al. Predictors and patterns of chronic pain three months after cardiac surgery in Taiwan. Pain Med. 2010;11(12):1849-58.

10. Chaparro LE, Smith SA, Moore RA, Wiffen PJ, Gilron I. Pharmacotherapy for the prevention of chronic pain after surgery in adults. Cochrane Database Syst Rev. 2013;7:CD008307.

11. Lahtinen P, Kokki H, Hynynen M. Pain after cardiac surgery: a prospective cohort study of 1-year incidence and intensity. Anesthesiology. 2006;105(4):794-800.

12. Lahtinen P, Kokki H, Hendolin H, Hakala T, Hynynen M. Propacetamol as adjunctive treatment for postoperative pain after cardiac surgery. Anesth Analg. 2002;95(4):813-9.

13. Dworkin RH, Kirkpatrick P. Pregabalin. Nat Rev Drug Discov. 2005;4(6):455-6.

14. Attal N, Cruccu G, Baron R, Haanpaa M, Hansson P, Jensen TS, et al. EFNS guidelines on the pharmacological treatment of neuropathic pain: 2010 revision. Eur J Neurol.2010;17(9):1113-e88.

15. Clarke H, Bonin RP, Orser BA, Englesakis M, Wijeysundera DN, Katz J. The prevention of chronic postsurgical pain using gabapentin and pregabalin: a combined systematic review and metaanalysis. Anesth Analg. 2012;115(2):428-42.

16. Apfelbaum JL, Hagberg CA, Caplan RA, Blitt CD, Connis RT, Nickinovich DG, et al. Practice guidelines for management of the difficult airway: an updated report by the American Society of Anesthesiologists Task Force on Management of the Difficult Airway. Anesthesiology. 2013;118(2):251-70.

17. Mellin-Olsen J, Staender S, Whitaker DK, Smith AF. The Helsinki Declaration on Patient Safety in Anaesthesiology. Eur J Anaesthesiol. 2010;27(7):592-7.

18. Huskisson EC. Measurement of pain.JRheumatol.1982;9(5):768-9.

19. Mahoori A, Noroozinia H, Hasani E, Hosainzadeh S. The effect of pre-operative administration of gabapentin on post-operative pain relief after herniorrhaphy. Saudi JAnaesth. 2014;8(2):220-3.

20. Mahoori A, Noroozinia H, Hasani E, Saghaleini H. Comparing the effect of pregabalin, gabapentin, and acetaminophen on postdural puncture headache. Saudi J Anaesth. 2014;8(3):374-7.

21. Joshi SS, Jagadeesh AM. Efficacy of perioperative pregabalin in acute and chronic post-operative pain after off-pump coronary artery bypass surgery: a randomized, double-blind placebo controlled trial. Ann Card Anaesth. 2013;16(3):180-5. 